\title{
Piriformospora indica Stimulates Root Metabolism of Arabidopsis thaliana
}

\author{
Nadine Strehmel ${ }^{1, *}$, Susann Mönchgesang ${ }^{1}$, Siska Herklotz ${ }^{1}$, Sylvia Krüger ${ }^{1}$, Jörg Ziegler ${ }^{2}$ and \\ Dierk Scheel ${ }^{1, *}$ \\ 1 Department of Stress and Developmental Biology, Leibniz Institute of Plant Biochemistry, Weinberg 3, \\ 06120 Halle (Saale), Germany; susann.moenchgesang@ipb-halle.de (S.M.); \\ siska.herklotz@pflanzenphys.uni-halle.de (S.H.); sylvia.krueger@ipb-halle.de (S.K.) \\ 2 Department of Molecular Signal Processing, Leibniz Institute of Plant Biochemistry, Weinberg 3, \\ 06120 Halle (Saale), Germany; joerg.ziegler@ipb-halle.de \\ * Correspondence: nstrehme@ipb-halle.de (N.S.); dierk.scheel@ipb-halle.de (D.S.); \\ Tel.: +49(0)-345-5582-1400 (D.S.)
}

Academic Editors: Ute Roessner and Jianhua Zhu

Received: 14 May 2016; Accepted: 28 June 2016; Published: 8 July 2016

\begin{abstract}
Piriformospora indica is a root-colonizing fungus, which interacts with a variety of plants including Arabidopsis thaliana. This interaction has been considered as mutualistic leading to growth promotion of the host. So far, only indolic glucosinolates and phytohormones have been identified as key players. In a comprehensive non-targeted metabolite profiling study, we analyzed Arabidopsis thaliana's roots, root exudates, and leaves of inoculated and non-inoculated plants by ultra performance liquid chromatography/electrospray ionization quadrupole-time-of-flight mass spectrometry (UPLC/(ESI)-QTOFMS) and gas chromatography/electron ionization quadrupole mass spectrometry (GC/EI-QMS), and identified further biomarkers. Among them, the concentration of nucleosides, dipeptides, oligolignols, and glucosinolate degradation products was affected in the exudates. In the root profiles, nearly all metabolite levels increased upon co-cultivation, like carbohydrates, organic acids, amino acids, glucosinolates, oligolignols, and flavonoids. In the leaf profiles, we detected by far less significant changes. We only observed an increased concentration of organic acids, carbohydrates, ascorbate, glucosinolates and hydroxycinnamic acids, and a decreased concentration of nitrogen-rich amino acids in inoculated plants. These findings contribute to the understanding of symbiotic interactions between plant roots and fungi of the order of Sebacinales and are a valid source for follow-up mechanistic studies, because these symbioses are particular and clearly different from interactions of roots with mycorrhizal fungi or dark septate endophytes
\end{abstract}

Keywords: plant; fungus; interaction; exudates; roots; leaves; metabolite profiling; liquid chromatography/mass spectrometry (LC/MS); gas chromatography/mass spectrometry (GC/MS)

\section{Introduction}

Piriformospora indica is a root-interacting endophytic fungus and has been found in the Indian Thar-Dessert [1]. It belongs to the order of Sebacinaceous (Basidiomycota) [2] and yields a growth promotion effect with various crop plants such as barley, tobacco, maize, and tomato, but also with the model plant Arabidopsis thaliana [3]. Previous studies showed that $P$. indica promotes nutrient uptake and helps plants to survive under biotic (pathogenic organisms) [4,5] and abiotic (water, temperature, salt, toxins, heavy metal ions) stress conditions [6,7]. Furthermore, it stimulates plant growth, biomass, and seed production [8,9]. The fungus colonizes the epidermal and rhizodermal part of the roots and forms pearshaped spores, which accumulate within the roots and on the root surface. P. indica grows inter- and intracellularly [10] but does not invade the endodermis and aerial parts of the plant. 
This endosymbiotic interaction has been considered as mutualistic, as it leads to an improved nutrient state in the host [11]. After establishment of this endosymbiotic interaction, the plant obtains more phosphorous and water through extracellular hyphae of the fungus, whereas the fungus is supplied with nitrogen and hydrocarbons in form of plant amino acids [11-15].

$P$. indica can be cultivated with the model plant $A$. thaliana. In general, $P$. indica colonization is host-dependent and occurs in two phases: Early interactions are biotrophic in barley and A. thaliana, but can switch to saprotrophy or maintain biotrophy in later stages, respectively [15]. Host metabolism determines the availability of nitrogen, and the subsequent induction of nitrogen transporters and a possible nutritional switch of $P$. indica from biotrophy to saprotrophy. A. thaliana had been shown to provide sufficient nitrogen sources in form of increased levels of amino acids like Gln and Asn at 14 dpi.

During the initial phase of this interaction, defense genes are activated and reactive oxygen species (ROS) produced by the host against $P$. indica [16]. However, $P$. indica can rescue plants with elevated ROS levels by providing antioxidants [17]. After recognition of $A$. thaliana, $P$. indica releases effectors into the rhizosphere, which induce a response in the host [18]. Moreover, an increase in the intracellular calcium concentration in the host's root cells is provoked, which triggers an intracellular signaling cascade (mitogen-activated protein kinase signaling pathways) $[19,20]$, whereupon ethylene signaling components and ethylene-response transcription factors are required [21,22]. Furthermore, cytokinins and auxins play a crucial role in the maintenance of this symbiotic interaction [23]. Lahrmann et al. [24] and others showed that the colonization of $A$. thaliana with $P$. indica correlates with the induction of salicylic acid catabolites and jasmonate as well as glucosinolate metabolism [25,26]. Indolics were identified as key players in the maintenance of this mutualistic interaction. Especially indolic glucosinolates and reaction products are required to restrict the growth of $P$. indica.

Since the genomes of both organisms have been completely sequenced, both partners offer an ideal opportunity to study mutualistic interactions of plants and root endophytes in the rhizosphere [27,28]. Thus, we investigated the metabolic response of $A$. thaliana to $P$. indica under hydroponic conditions by non-targeted liquid chromatography/mass spectrometry (LC/MS) and gas chromatography/mass spectrometry (GC/MS)-based metabolite profiling. For this purpose, we chose ultra performance liquid chromatography coupled to electrospray ionization quadrupole time-of-flight mass spectrometry (UPLC/ESI-QTOFMS) for the profiling of secondary metabolites and gas chromatography coupled to electron ionization quadrupole mass spectrometry (GC/EI-QMS) for the profiling of primary metabolites. Both platforms gain a snapshot of biochemical processes within a cell. Whereas reversed-phase LC/MS allows for the profiling of semipolar compounds [29], namely indolics, flavonoids, phenylpropanoids, glucosinolates and their degradation products, GC/EI-QMS covers main parts of central carbon metabolism [30]. Regardless of the choice of analysis platform, all samples can be grouped according to their common metabolic fingerprint. For this purpose we set up a standardized co-cultivation system, which supports the growth of both partners in close association to each other and the consequent profiling of roots and their exudates as well as leaves.

\section{Results and Discussion}

To study the interaction of $P$. indica with $A$. thaliana, a sterile hydroponic cultivation system was developed, which allows for the simultaneous profiling of roots and their exudates (Supplementary Figure S1). For this purpose, P. indica was precultivated on agar plates and A. thaliana on agar-filled, bottom-cut PCR tubes protruding into a liquid culture medium. After two weeks, both organisms were brought together in half-strength Murashige-Skoog (MS) medium supplemented with $0.5 \%$ sucrose $(w / v)$ and Gamborg B5 vitamins such as myo-inositol, nicotinic acid, pyridoxin, and thiamine. According to our preliminary studies both components are essential for this symbiosis and hence the growth promotion effect of $A$. thaliana. 
2.1. P. indica Promotes Shoot Growth of A. thaliana under Specific Culturing Conditions in a Hydroponic System after Root Colonization

If both components (sucrose and Gamborg B5 vitamins) were supplied for the co-cultivation studies, the shoot biomass increased by $22 \%\left(p=4.2 \times 10^{-5}\right)$ in inoculated samples compared to the control confirming the previously reported growth promotion effect in soil [31]. Although P. indica colonizes the roots, the root biomass did not change after two weeks of co-cultivation (Figure 1) leading to the assumption that $P$. indica might provoke a systemic effect in A. thaliana. Although previous studies have shown a growth promotion effect in roots [23,31], we anticipated slight deviations in a hydroponic system compared to soil due to different physicochemical properties.

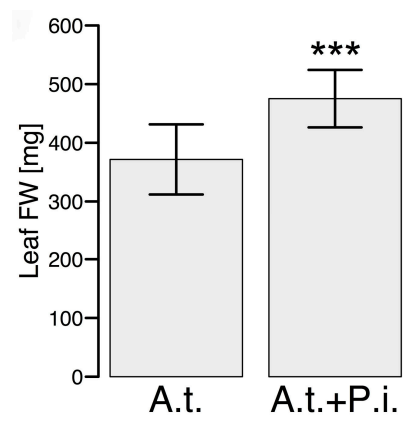

(A)

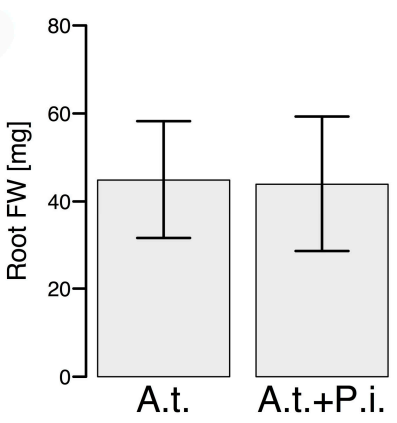

(B)

Figure 1. Leaf and root fresh weight of $A$. thaliana (A.t.) after co-cultivation with P. indica (P.i.) in a hydroponic system. A. thaliana was co-cultivated for two weeks with an agar plug containing mycelia of $P$. indica. For control $A$. thaliana was solely cultivated with an agar plug in $0.5 \times$ Murashige \& Skoog (MS) medium supplemented with $0.5 \%$ sucrose $(w / v)$ and vitamins: (A) shoot fresh weight (FW); (B) root fresh weight $(\mathrm{FW})$. Values represent the mean \pm SD (standard deviation) of three independent experiments (control samples: $n=3 \times(3-5)$ and co-cultivated samples: $n=3 \times 5$ ). Each replicate $n$ comprises a pool of 24 plants. Significance analysis of differences between control and co-cultivated samples was performed by $t$-test: ${ }^{* *}, p \leqslant 0.001$.

To investigate how $P$. indica interacts with the host in a hydroponic system, fluorescence microscopy images were recorded using green fluorescent protein (GFP)-labeled P. indica and the interaction monitored at $14 \mathrm{dpi}$.

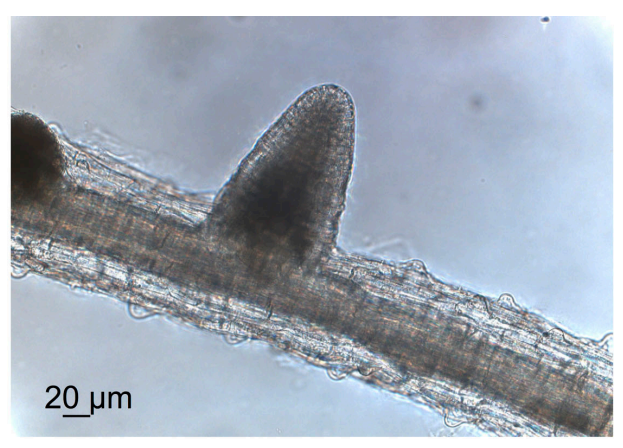

(A)

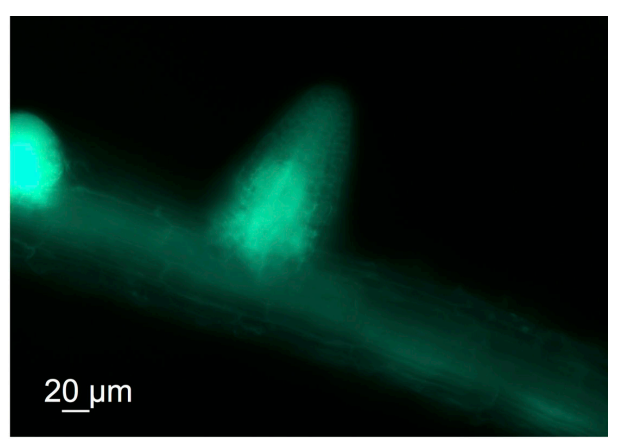

(B)

Figure 2. Microscopic image of an inoculated root with a GFP-labeled P. indica strain. (A) brightfield image; (B) fluorescence image.

P. indica grows inter- and intracellularly in root cells of $A$. thaliana when co-cultivated in soil [31]. In order to test if $P$. indica still forms fungal hyphae at the root surface under hydroponic conditions, a GFP-labeled $P$. indica strain was used to visualize colonization. Only weak autofluorescence signals 
were detected in the non-inoculated roots and roots inoculated with the non-labeled P. indica strain (Supplementary Figure S2). In contrast, roots inoculated with the GFP-labeled strain exhibited a very strong fluorescence already after a $3 \mathrm{~s}$ exposure time showing that $P$. indica colonizes the root surface and penetrates the root of $A$. thaliana (Figure 2). Interestingly, P. indica was predominantly detected in lateral roots. According to these observations, we concluded that $P$. indica colonizes roots of $A$. thaliana and as a consequence likely leads to changes in root and shoot metabolism. So far, only indolic glucosinolates and hormones have been discussed in depth [24,26].

\subsection{P. indica Alters the Exudation of Secondary Metabolites by A. thaliana Roots}

Hormonal regulation has been described to accompany the colonization of $P$. indica on A. thaliana roots [22-24,32-35]. An enrichment analysis (Table 1 and Supplementary Table S1) of the upregulated root transcripts $14 \mathrm{dpi}$ as published in Lahrmann et al. [24] revealed an overrepresentation of genes involved in the Kyoto Encyclopedia of Genes and Genomes (KEGG) pathway “Biosynthesis of plant hormones" (ath01070).

Table 1. Overrepresented KEGG pathways among upregulated A. thaliana root transcripts 14 dpi [15].

\begin{tabular}{lcc}
\hline \multicolumn{1}{c}{ Term } & Fold Enrichment & $\boldsymbol{p}$-Value * \\
\hline ath00966: Glucosinolate biosynthesis & 10.4 & $8.89 \times 10^{-8}$ \\
ath00940: Phenylpropanoid biosynthesis & 3.8 & $5.84 \times 10^{-7}$ \\
ath00360: Phenylalanine metabolism & 3.7 & $6.21 \times 10^{-5}$ \\
ath00903: Limonene and pinene degradation & 3.8 & $1.12 \times 10^{-4}$ \\
ath00680: Methane metabolism & 3.5 & $1.70 \times 10^{-4}$ \\
ath00945: Stilbenoid, diarylheptanoid and gingerol biosynthesis & 3.7 & $2.20 \times 10^{-4}$ \\
ath00910: Nitrogen metabolism & 3.9 & $5.56 \times 10^{-3}$ \\
ath00260: Glycine, serine and threonine metabolism & 3.7 & $7.07 \times 10^{-3}$ \\
ath00460: Cyanoamino acid metabolism & 5.0 & $1.25 \times 10^{-2}$ \\
ath00960: Tropane, piperidine and pyridine alkaloid biosynthesis & 5.5 & $2.05 \times 10^{-2}$ \\
ath01070: Biosynthesis of plant hormones & 1.6 & $3.54 \times 10^{-2}$ \\
ath00400: Phenylalanine, tyrosine and tryptophan biosynthesis & 3.3 & $4.44 \times 10^{-2}$ \\
\hline
\end{tabular}

* $p$-value was corrected according to Benjamini-Hochberg.

As shown in Supplementary Figure S3, $P$. indica significantly affects phytohormone levels in root exudates and roots, respectively. A higher concentration of hormones was found in exudates of co-cultivated samples as compared to control samples. This effect was in particular pronounced for jasmonate (JA), and jasmonyl-isoleucine (JA-Ile), both showing a more than 10-fold increase in the exudates and its potential role was discussed in reference [24]. In roots, the hormone content was also increased, but to a lower extent for JA, and JA-Ile, for which only a two- to four-fold increase was observed. 12-oxo-phytodienoic acid (OPDA), the precursor of JA and JA-Ile, also accumulated in roots but could not be detected in exudates irrespective of the conditions.

Besides the transcriptional regulation of hormone biosynthesis, hormone responses were overrepresented biological processes in Gene Ontology (GO:0009753 response to jasmonic acid stimulus, GO:0009751 response to salicylic acid stimulus). The analysis of Gene Ontology (GO) terms (Supplementary Table S1) further pointed to the involvement of secondary metabolic processes as the top two enriched processes (GO:0019748) ranked after defense response (GO:0006952). Consequently, roots and their exudates were comprehensively profiled for changes in primary and secondary metabolism upon P. indica colonization. Root exudates were only profiled for changes in semipolar metabolism, as in a screen for primary metabolites (GC/MS) all blank samples (samples without plant and/or fungus) already exhibited a considerable number of primary metabolites. Representative base peak chromatograms are depicted in Figure 3 and reveal a unique metabolic fingerprint for both ionization modes, ESI(+) and ESI(-). A principal component analysis (PCA) could verify this 
assumption. For both ionization modes $89 \%$ of the total variance was explained by the first principal component (PC1) and 3\% ESI(+) as well as 4\% ESI(-) by PC2 (Supplementary Figure S4).

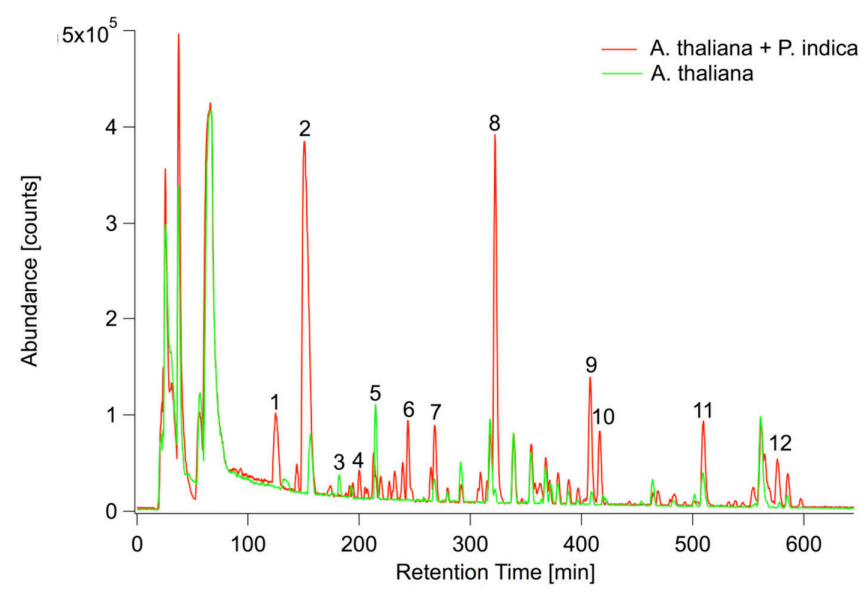

(A)

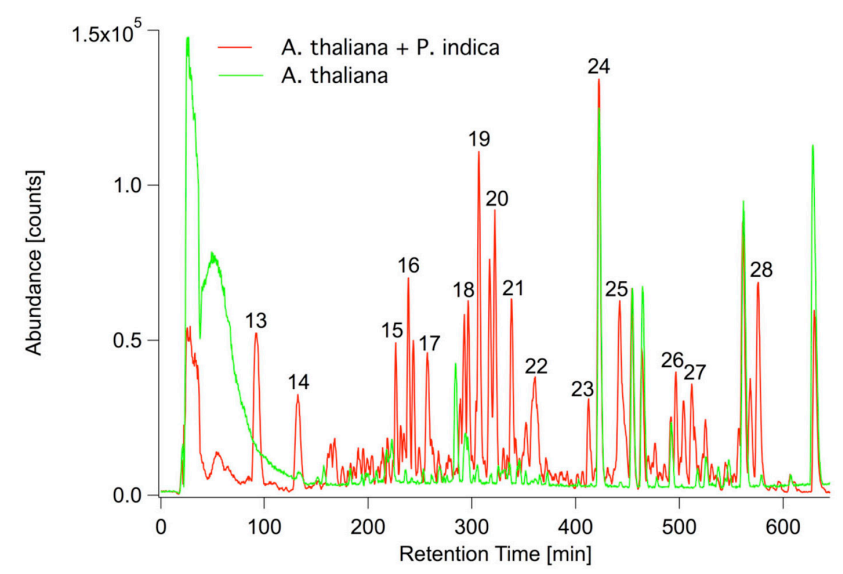

(B)

Figure 3. Overlay of representative UPLC/ESI(+/-)-QTOFMS base peak chromatograms $(\mathrm{m} / \mathrm{z}$ 100-1000) of inoculated (red) and non-inoculated (green) A. thaliana exudates. (A) ESI(+): positive ionization mode; (B) ESI(-): negative ionization mode. 1: 8-MeSO-Octyl- $\mathrm{NH}_{2} ; 2: \mathrm{C}_{10} \mathrm{H}_{15} \mathrm{~N}_{3}$; 3: $\mathrm{H}-\mathrm{Ile}-\mathrm{Ile}-\mathrm{OH} ;$ 4: 1-MeO-I3 $\mathrm{CH}_{2} \mathrm{NH}_{2} ; \quad$ 5: $\mathrm{C}_{9} \mathrm{H}_{7} \mathrm{~N}_{3} \mathrm{O}_{3} ;$ 6: $\mathrm{C}_{17} \mathrm{H}_{34} \mathrm{NO}_{9} \mathrm{P} ;$ 7: Scopoletin; 8: 8-MeSO-Octyl-CN; 9: $\mathrm{C}_{16} \mathrm{H}_{29} \mathrm{NO}_{8} ;$ 10: $\mathrm{C}_{12} \mathrm{H}_{20} \mathrm{O}_{4} ; 11: \mathrm{C}_{14} \mathrm{H}_{28} \mathrm{O}_{5} ; 12: \mathrm{C}_{28} \mathrm{H}_{42} \mathrm{O}_{6} ;$ 13: Pantothenic acid; 14: $\mathrm{C}_{16} \mathrm{H}_{26} \mathrm{O}_{8} ; 15: \mathrm{C}_{16} \mathrm{H}_{23} \mathrm{~N}_{3} \mathrm{O}_{8} ; 16: \mathrm{C}_{16} \mathrm{H}_{23} \mathrm{~N}_{3} \mathrm{O}_{8} ; 17: \mathrm{C}_{13} \mathrm{H}_{24} \mathrm{O}_{6} ; 18: \mathrm{C}_{12} \mathrm{H}_{22} \mathrm{O}_{5} ; 1$ 19: $\mathrm{C}_{9} \mathrm{H}_{18} \mathrm{O}_{4} ; 20: \mathrm{C}_{13} \mathrm{H}_{22} \mathrm{O}_{5} ; 21: \mathrm{C}_{12} \mathrm{H}_{22} \mathrm{O}_{5} ; 22: \mathrm{C}_{25} \mathrm{H}_{41} \mathrm{~N}_{3} \mathrm{O}_{9} ; 23: \mathrm{C}_{14} \mathrm{H}_{26} \mathrm{O}_{5} ; 24$ : Internal standard 2,4-Dichlorophenoxyacetic acid; 25: 9,12,13-Trihydroxyoctadec-10-enoic acid; 26: $\mathrm{C}_{12} \mathrm{H}_{18} \mathrm{O}_{4} ;$ 27: $\mathrm{C}_{28} \mathrm{H}_{44} \mathrm{O}_{6} ; 28: \mathrm{C}_{28} \mathrm{H}_{42} \mathrm{O}_{6}$.

Non-targeted UPLC/ESI(+/-)-QTOFMS-based metabolite profiling revealed that the concentration of 200 out of 341 detected ESI(+) components as well as 271 out of 377 ESI(-) components was significantly affected $(p<0.01)$ due to the presence of $P$. indica. A total of $28(\mathrm{ESI}(+))$ as well as $24(\operatorname{ESI}(-))$ components were down- and $172(\operatorname{ESI}(+))$ as well as $247(\operatorname{ESI}(-))$ components were upregulated due to the inoculation implying that $P$. indica stimulates root exudation of $A$. thaliana.

As already observed by Lahrmann et al. [24], the amount of compounds associated with nucleoside and aromatic amino acid metabolism was reduced in concentration by the inoculation, while that of aliphatic and indolic glucosinolate metabolism (except for 4-hydroxy-indole-3-carbaldehyde), dihydroxybenzoic acid (DHBA) conjugates, JA metabolism as well as fatty acid and pantothenic acid metabolism was increased. A number of phenylpropanoids including coumarins and oligolignols 
(except for scopoletin and G(8-O-4)FA sulfate) showed reduced levels in the exudates of inoculated samples (Figure 4) leading to the assumption that these oligomers are further metabolized inside the cell and not exuded, very likely to oligolignols or to lignin [36], a main constituent of the cell wall. Both, glucosinolate (ath00966) and phenylpropanoid biosynthesis (ath00940), were among the overrepresented KEGG pathways of root transcripts (Table 1).

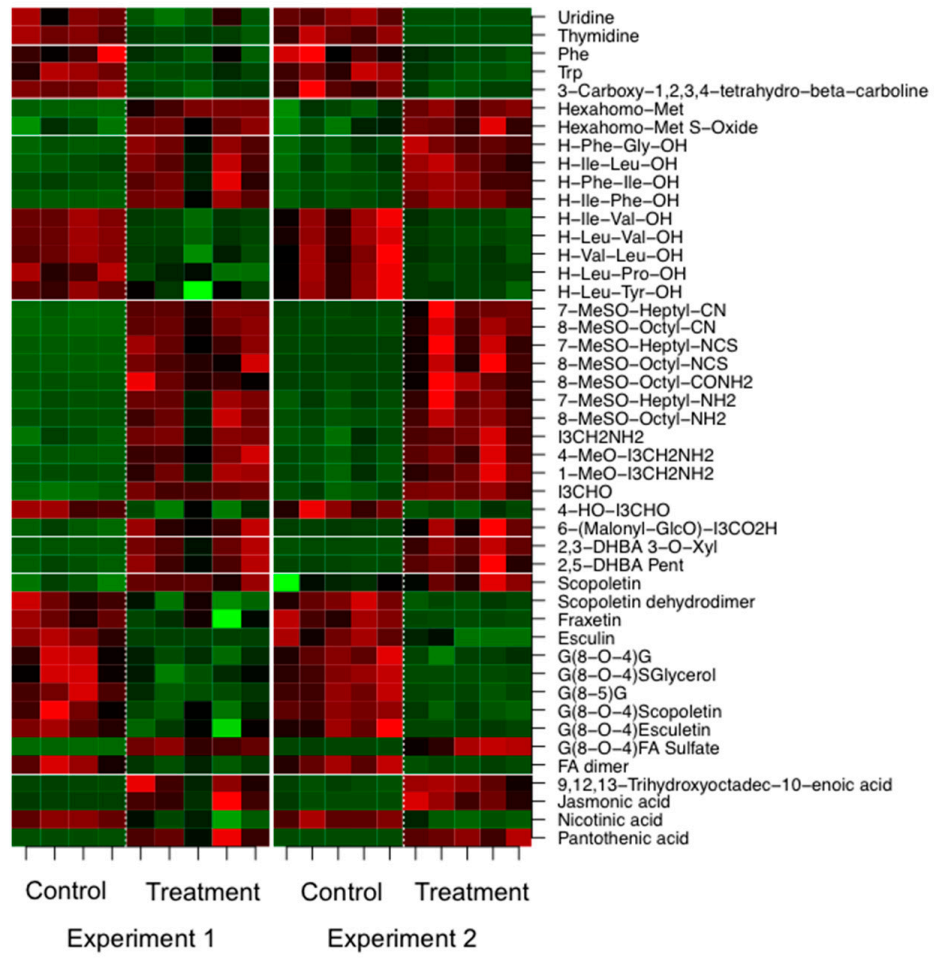

Figure 4. Differentially expressed metabolites $(p \leqslant 0.01)$ in root exudates of $A$. thaliana after co-cultivation with the fungus $P$. indica for two weeks across two independent biological experiments. Intensity values were log-transformed and z-scored row-wise. Red: maximal intensity; Green: minimal intensity.

Nicotinic acid, an important precursor for vitamin B6, and thus, key player in the photoprotection of plants [37], also decreased in concentration upon co-cultivation in the root exudates (Figure 4). Obviously, nicotinic acid is required by $P$. indica. If this compound was not supplemented, no growth-promoting effect was observed of the host.

In the exudates we also detected differences in the dipeptide pool, namely the concentration of Phe-Gly, Ile-Leu, Phe-Ile and Ile-Phe was enhanced, while that of Ile-Val, Leu-Val, Val-Leu, Leu-Pro and Leu-Tyr was reduced in the co-cultivated samples (Figure 4). These differences might originate from different functionalities of the respective dipeptides and require further investigation. So far, Komarova et al. [38] showed that peptide transporters (AtPTR5 and AtPTR5) facilitate the uptake of nitrogen from the rhizosphere.

\subsection{Changes in the Root Metabolism of A. thaliana}

The secondary metabolic changes detected in root exudates, especially that of glucosinolate biosynthesis, phenylpropanoid biosynthesis, and phenylalanine metabolism should also be reflected in root metabolism. In addition, transcriptionally enriched KEGG pathways of primary metabolism (Table 1), such as nitrogen metabolism (ath00910), glycine, serine and threonine metabolism (ath00260), and cyanoamino acid metabolism (ath00460) were expected in the GC/MS-based metabolite profiles. 
2.3.1. Non-Targeted GC/MS Based Metabolite Profiling Reveals Perturbations in the Primary Root Metabolism

Figure 5 shows two representative extracted ion chromatograms of $m / z 73$ (equals $\mathrm{C}_{3} \mathrm{H}_{9} \mathrm{Si}^{+}$ and is a typical fragment for trimethylsilylated compounds) obtained from a pool of inoculated and non-inoculated roots. Again, the inoculated profile is distinct compared to the non-inoculated root metabolic profile. Forty-eight percent of the total variance was explained by PC1 and 13\% by PC2 and are plotted in Supplementary Figure S5.

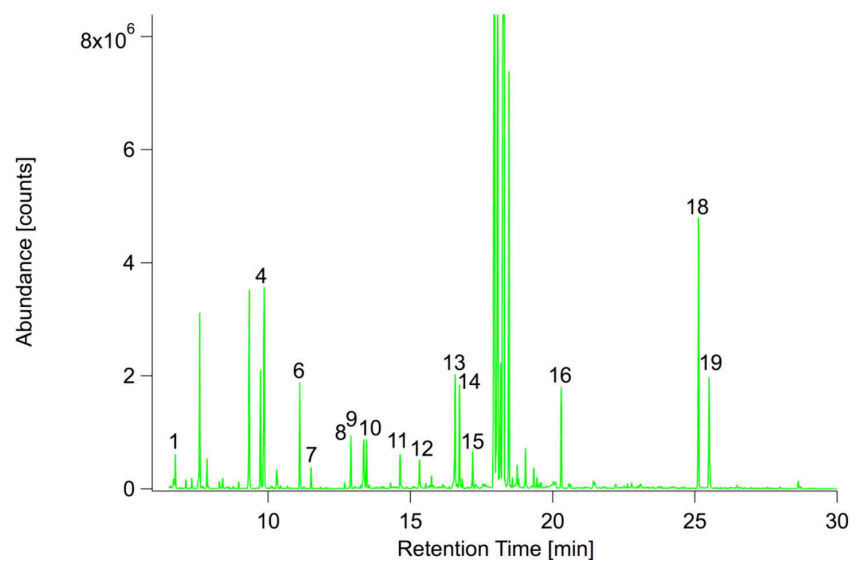

(A)

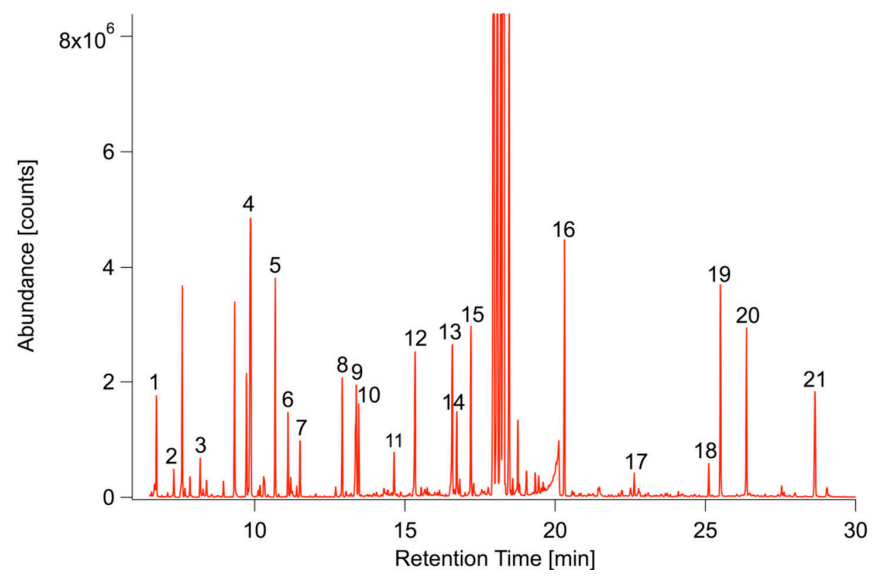

(B)

Figure 5. Representative extracted ion chromatograms $(m / z 73)$ of inoculated and non-inoculated A. thaliana root extracts. (A) Non-inoculated root; (B) with P. indica inoculated root. 1: Lactic acid (2TMS); 2: Alanine (2TMS); 3: Sulfuric acid (2TMS); 4: Phosphoric acid (3TMS); 5: Glyceric acid (3TMS); 6: Serine (3TMS); 7: Threonine (3TMS); 8: Malic acid (3TMS); 9: Pyroglutamic acid (2TMS); 10: GABA (3TMS); 11: Glutamic acid (3TMS); 12: Asparagine (3TMS); 13: Glutamic acid (3TMS); 14: Glutamine (3TMS); 15: Citric acid (4TMS); 16: Myo-Inositol (6TMS); 17: Glucose-6-phosphate (1MeOX, 6TMS); 18: Thiamine hexoside; 19: Sucrose (8TMS); 20: Unknown; 21: Unknown.

Non-targeted GC/EI-Q-MS based metabolite profiling revealed 287 out of 801 differentially accumulated components. Among them, we detected amino acids (e.g., Asn, Thr, Leu, 3-Cyano-Ala, beta-Ala, Val, Ala, Gln, ornithine, Pro, pyro-Glu, and GABA), organic acids (e.g., citrate, 2-oxoglutarate, fumarate, malate, oxalate, glycerate, fumarate, and 3-hydroxy-3-methylglutaric acid), carbohydrates (e.g., 1-O-methylglucopyranoside, 1-O-methylgalactopyraoside, maltose, raffinose, trehalose, xylose, ribose), polyols (erythritol, myo-inositol), phosphates (e.g., glycerol-3-phosphate, phosphate, glycerophosphoglycerol), and sulfates (e.g., sulfate, thiamine, thiamine-hex) belonging to the starch 
and sucrose metabolism, glycolysis, tricarboxylic acid (TCA) cycle, amino acid metabolism, and urea metabolism. All compounds showed increased levels in the inoculated roots (Figure 6) except for pyruvate, erythritol, allantoin, and 4-methyl-5-thiazoleethanolglycopyranoside (for spectrum see Supplementary Figure S6) indicating that the initially applied amount of sucrose and thiamine is metabolized by P. indica. We observed an increase in the concentration of Asn, Gln, Ser, Thr, and Ala at $14 \mathrm{dpi}$. Serine was also increased in its levels as described by Lahrmann et al. [15], but did not pass the defined significance level $(p=0.051)$. In general, the data collected are in good accordance with the transcriptional changes and lead us to the hypothesis that $A$. thaliana provides nitrogen to the fungus so that $P$. indica can maintain a biotrophic nutritional state [15]. In the leaf profiles, the $\mathrm{N}$-rich amino acids (Gln, Arg, Asn, 3-Cyano-Ala, and ornithine) were among the few differentially accumulated compounds decreasing in concentration upon colonization and consequently showed the opposite trend (Supplementary Figure S7) compared to the roots. This raises the question if these amino acids are transported to the root to feed $P$. indica. Most likely, these amino acids are required to balance the nutritional state of $P$. indica. To trace the flow of nutrients, further investigations are required. The change in the concentration of organic acids and carbohydrates was comparable for roots and leaves except that less differential changes were observed in the leaf profiles. These results again show that $P$. indica activates primary root metabolism of $A$. thaliana. According to our data, both partners appear to offer each other nutrients to maintain a mutualistic interaction, since an enhanced amount of $\mathrm{P}, \mathrm{S}$, and $\mathrm{N}$ (in the form of amino acids) was observed in roots of $A$. thaliana colonized with P. indica.

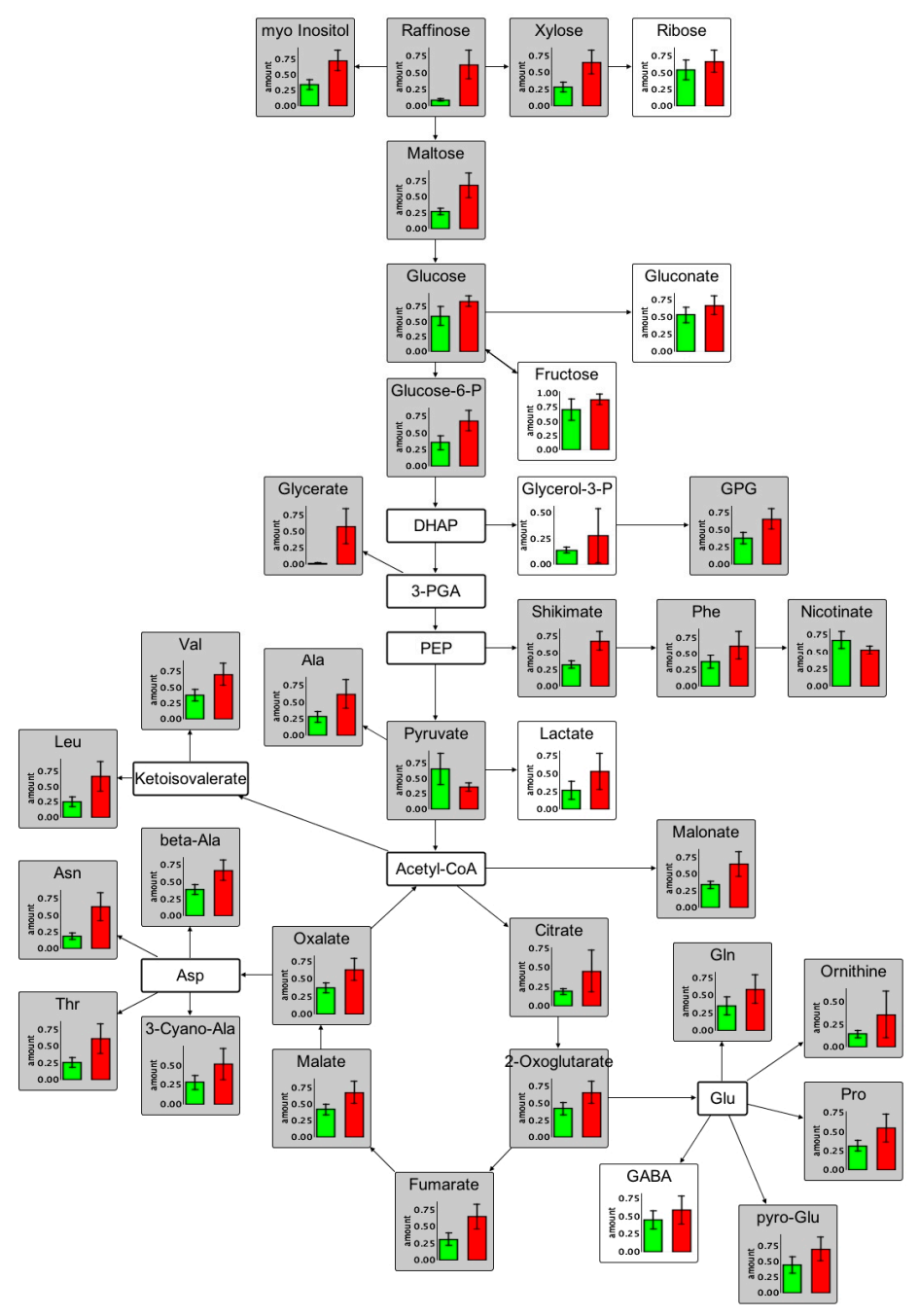

Figure 6. Cont. 


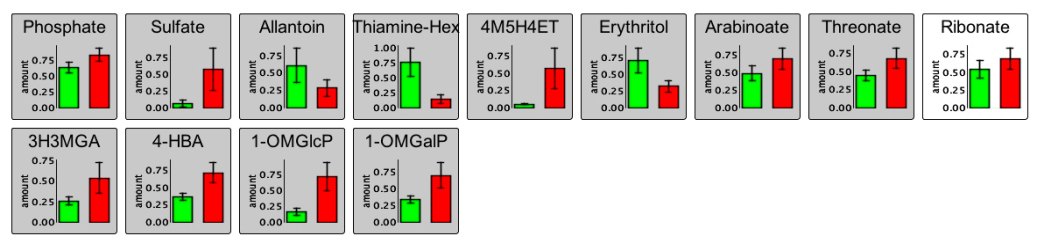

Figure 6. Differentially expressed primary metabolites occurring in root extracts of $A$. thaliana. Control and treatments are color-coded; control: A. thaliana (green) and treatment: A. thaliana + $P$. indica (red). Compounds with $p<0.01$ are specifically marked by grey color or left blank for $0.01 \leqslant p \leqslant 0.05$. GPG: glycerophosphoglycerol; 4M5HET: 4-methyl-5-hydroxyethylthiazole; 3H3MGA: 3-hydroxy-3-methylglutaric acid; 4-HBA: 4-hydroxybenzoic acid; 1-OMGclP: 1-O-methyl-glucopyranoside; 1-OMGalP: 1-O-methylgalactopyranoside.

2.3.2. LC/MS Based Non-Targeted Metabolite Profiling Shows an Induction of Aliphatic and Indolic Glucosinolate Metabolism, Flavonoids, and Oligolignols in Roots

Besides primary metabolism, secondary root metabolism was investigated, since one category "secondary metabolic process" was a highly ranked candidate in the GO enrichment analysis. A unique fingerprint was observed in the root LC/MS profiles (Figure 7). According to Supplementary Figure S5, 76\% of the entire variance was explained by PC1 and $0.07 \%$ by PC2 for the positive mode. These values were similar for the negative mode (PC1: 74\%; PC2: 0.08\%) and indicate that secondary metabolism is perturbed to a greater extent than primary metabolism.

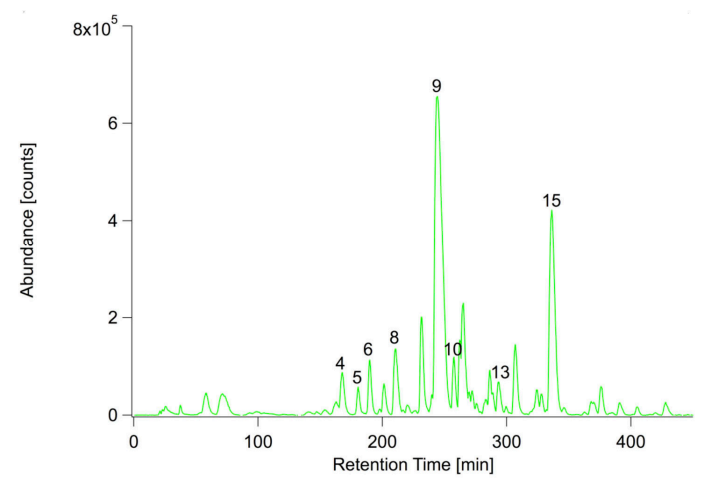

(A)

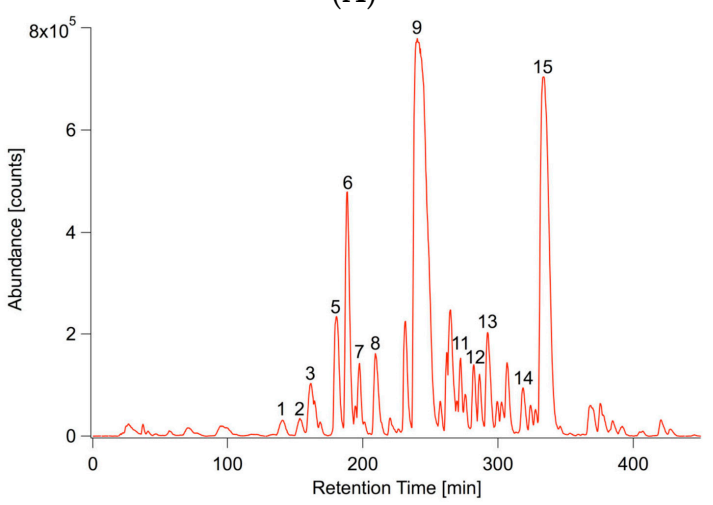

(B)

Figure 7. Representative UPLC/ESI(-)-QTOFMS base peak chromatograms $(m / z$ 100-1000) of inoculated and non-inoculated $A$. thaliana root extracts. (A) Non-inoculated root; (B) with $P$. indica inoculated root. 1: 7MeSO Heptyl GSL; 2: 2,5 DHBA-Pent; 3: I3M GSL; 4: $\mathrm{C}_{14} \mathrm{H}_{18} \mathrm{O}_{10} ; 5: \mathrm{C}_{17} \mathrm{H}_{24} \mathrm{O}_{10}$; 6: $8 \mathrm{MeSO}$ Octyl GSL; 7: Scopolin; 8: 4MeO-I3M GSL 9: 1MeO-I3M GSL; 10: $\mathrm{C}_{18} \mathrm{H}_{32} \mathrm{O}_{11} ; 11$ : $\mathrm{C}_{19} \mathrm{H}_{18} \mathrm{O}_{3}$; 12: $\mathrm{C}_{19} \mathrm{H}_{8} \mathrm{O}_{3} ;$ 13: 7MeS Heptyl GSL; 14: $\mathrm{C}_{38} \mathrm{H}_{46} \mathrm{O}_{18} ; 15: 8 \mathrm{MeS}$ Octyl GSL. 
In these profiles, 167 out of 329 detected compounds $(\mathrm{ESI}(+))$ were altered in abundance and 188 out of 359 for the negative ionization mode due to the presence of $P$. indica. Similarly to the exudates, a higher number of compounds displayed upregulated abundance in the inoculated samples compared to the non-inoculated samples. From these numbers one can once more conclude that P. indica stimulates secondary root metabolism as well.

In accordance with Lahrmann et al. [24], aliphatic and indolic glucosinolates as well as their breakdown products, aromatic amino acids, coumarins, oligolignols, and flavonoids accumulated in inoculated roots (Figure 8) confirming the transcript data (KEGG, Table 1: glucosinolate ath00966 and phenylpropanoid biosynthesis ath00940). Although the plant seems to be in a defensive stage, no camalexin was detected in these profiles. In the leaf profiles an increased amount of aliphatic and indolic glucosinolates as well as their breakdown products, JA conjugates, oligolignols, and hydroxycinnamic acid amides was detected (Supplementary Figure S8). Several flavonoids (glycosylated kaempferol and quercetin) were only detected as differential in the root profiles and not in the leaf profiles, leading to the conclusion that this substance class plays an important role in the mutualistic interaction of $A$. thaliana and P. indica. Recently, Lahrmann et al. [24] stated that it remains to be clarified if flavonoids are accumulating in roots of $A$. thaliana upon interaction with $P$. indica. Indeed, we show that flavonoids accumulate in roots of $A$. thaliana upon co-cultivation with $P$. indica. Most likely, enhanced flavonoid biosynthesis, in addition to JA signaling [39], may also function as a signal for $P$. indica.

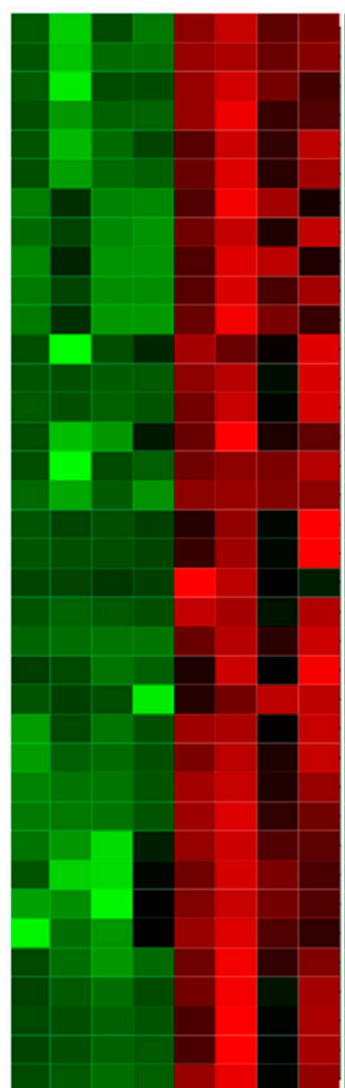

Control Treatment

Experiment 1

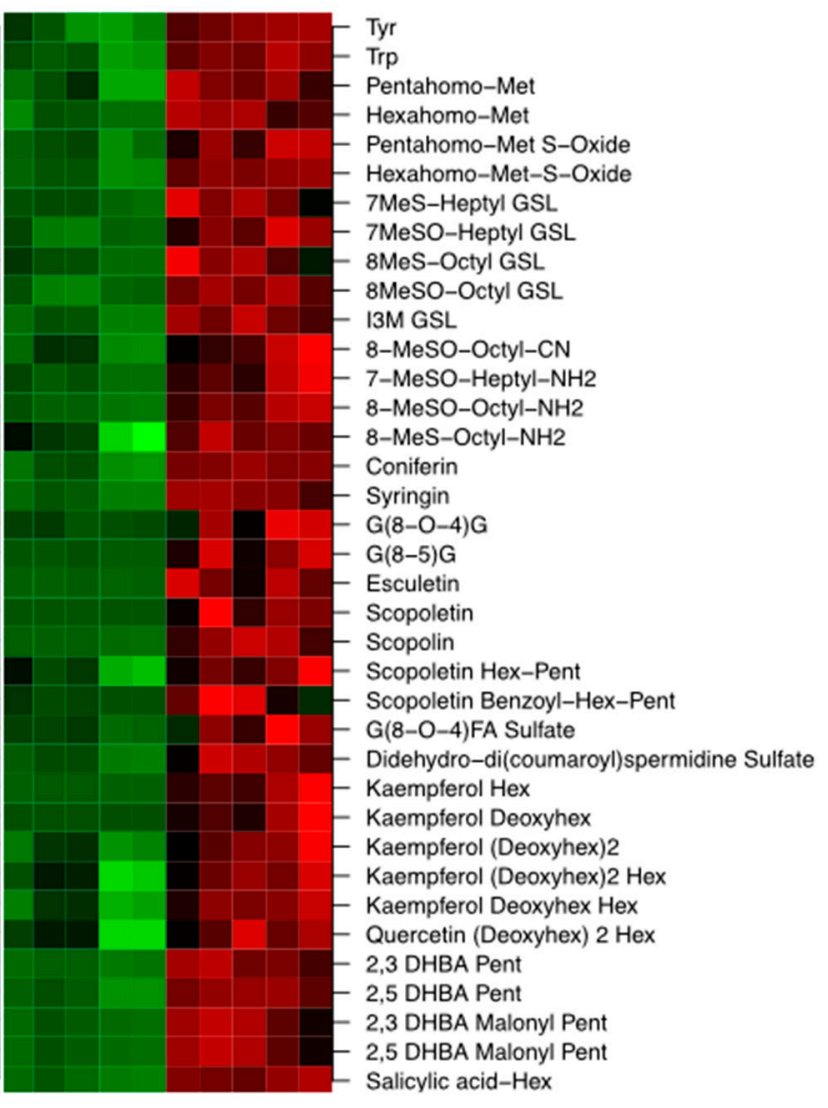

Control Treatment

Experiment 2

Figure 8. Differentially expressed secondary metabolites occurring in root extracts of $A$. thaliana across two independent biological experiments. Candidates were retrieved from a two-sided $t$-test $(p<0.01)$. For visualization, intensity values were log-transformed and $z$-scored row-wise. Red: maximal intensity; green: minimal intensity. 


\section{Materials and Methods}

\subsection{Chemicals and Standards}

All chemicals were of highest analytical grade (>99\%) and obtained from Carl Roth GmbH + Co. KG (Karlsruhe, Germany), Difco Microbiology (Lawrence, KS, USA), Duchefa Biochemie B.V. (Haarlem, The Netherlands), Merck KGaA (Darmstadt, Germany), and Sigma-Aldrich (Steinheim, Germany).

\subsection{Pre-Cultivation of P. indica}

P. indica was cultured on agar plates $\left(1.5 \%(w / v)\right.$ agar) for 3 weeks at $28{ }^{\circ} \mathrm{C}$ in the dark using Aspergillus minimal medium [29]. For this purpose, a punched out agar block with mycelia of $P$. indica was placed in the center of a culture plate.

\subsection{Conduction of Co-Cultivation Studies and Production of Plant Material}

Co-cultivation studies were performed as previously described [25]. In short, two-week old A. thaliana plantlets were co-cultivated for two weeks with $P$. indica in a hydroponic system under short day conditions $\left(23{ }^{\circ} \mathrm{C}, 8 \mathrm{~h}\right.$ light, $180 \mu \mathrm{E} \cdot \mathrm{m}^{-2} \cdot \mathrm{s}^{-1}$ and $21{ }^{\circ} \mathrm{C}, 16 \mathrm{~h}$ dark). After two weeks of co-cultivation (four-week old plants), the medium containing the nutrient solution and the root exudates was filtered and stored at $4{ }^{\circ} \mathrm{C}$ in Schott flasks until further processing. At harvest, roots were cut below the bottom of the PCR tube and blotted dry with a paper towel before shock freezing in liquid nitrogen. Finally, they were stored at $-80^{\circ} \mathrm{C}$ until further processing. More technical details are visualized in Supplementary Figure S1. Media composition is summarized in Appendix A.

\subsection{LC/MS-Based Metabolite Profiling}

For LC/MS-based metabolite profiling (UPLC: Acquity, Waters, Eschborn, Germany; MS: MicrOTOF-Q I hybrid quadrupole time-of-flight mass spectrometer equipped with an Apollo II electrospray ion source, Bruker Daltonik $\mathrm{GmbH}$, Bremen, Germany), the ground tissue material was processed by solid liquid extraction using methanol/water, 80/20 (v/v) (40 mg root fresh weight corresponds to $200 \mu \mathrm{L}$ extraction solution and $50 \mathrm{mg}$ leaf fresh weight corresponds to $400 \mu \mathrm{L}$ extraction solution). Analytes of the nutrient solution were extracted by a reversed-phase solid phase extraction procedure (180 mL medium result in $120 \mu \mathrm{L}$ analysis solution).

\subsubsection{Preparation of Nutrient Solutions for LC/MS Analysis}

All exudate samples were prepared and analyzed by UPLC/ESI-QTOFMS as presented in Lahrmann et al. [24]. In short, the nutrient solution was spiked with $20 \mu \mathrm{M} 2-(2,4$-dichlorophenoxy) acetic acid, evaporated until dryness, reconstituted in $9 \mathrm{~mL}$ water/methanol 95/5 (v/v) and subjected to a Bond Elut PPL cartridge (200 mg, $3 \mathrm{~mL}$, Agilent Technologies, Böblingen, Germany). Finally, the eluate was subjected to a solid phase extraction workup and reconstituted in $120 \mu \mathrm{L}$ water/methanol $70 / 30(v / v)$ prior to LC/MS analysis. Technical details of the solid phase extraction workup can be found in the Appendix B.

\subsubsection{Sample Preparation and Profiling of Tissue Material for LC/MS Analysis}

The plant material was processed according to Böttcher et al. [29]. As already described, the frozen material was extracted twice with methanol/water, 80/20 (v/v) and reconstituted in methanol/water, $30 / 70(v / v)$ prior to LC/MS-analysis. More details of the extraction procedure can be found in the Appendix B.

\subsubsection{Non-Targeted LC/MS-Based Profiling and Data Analysis}

Changes in the secondary plant metabolism were analyzed by UPLC/ESI-QTOFMS. Samples were injected onto an Acquity UPLC system (Waters, Eschborn, Germany), equipped with an HSS 
T3 column $(100 \times 1.0 \mathrm{~mm}$, particle size $1.8 \mu \mathrm{m}$, Waters $)$, and separated using a binary gradient (A: water $/ 0.1 \%(v / v)$ formic acid; B: acetonitrile $/ 0.1 \%(v / v)$ formic acid). Eluting compounds were detected in positive and negative ionization mode from $m / z$ 100-1000 using a MicroTOF-Q I hybrid quadrupole time-of-flight mass spectrometer equipped with an Apollo II electrospray ion source (Bruker Daltonics, Billerica, MA, USA). All instrument parameters and further settings can be found in the Appendix B.

Raw data files were converted to mzData using CompassXPort version 1.3.10 (Bruker Daltonics). For feature detection, alignment, and filling of missing values the $\mathrm{R}$ package XCMS version 1.41.0 [40] was used. Settings are summarized in Appendix B.

The intensities of the resulting features $\left(\mathrm{m} / \mathrm{z}\right.$-retention time pairs) were $\log _{2}$ transformed and subjected to a two-sided Student's $t$-test. Relevant mass spectral features were extracted within a predefined range (isolation width: $\pm 0.02 \mathrm{~m} / z$ ) and elemental compositions were calculated applying a default error range (15 ppm). Putative elemental compositions were checked for consistency while analyzing elemental compositions of fragment ions and neutral losses of collision-induced dissociation (CID)-mass spectra. For acquisition of CID mass spectra quasi-molecular cluster ions were isolated at the Q1 (isolation width: $\pm 3 \mathrm{~m} / \mathrm{z}$ ) and fragmented inside the collision cell using argon as collision gas. Product ions were detected as described above. All mass spectral data can be found in the MetaboLights repository (MTBLS341) [41].

\subsection{GC/MS Based Metabolite Profiling}

\subsubsection{Sample Preparation of Tissue Material}

One hundred $\mu \mathrm{L}$ extract of the remainder from the LC/MS-based metabolite profiling studies was spiked with $100 \mu \mathrm{M}$ succinic acid-2,2,3,3- $\mathrm{d}_{4}$, dried down in a vacuum concentrator, and stored at $-20^{\circ} \mathrm{C}$ until further processing.

\subsubsection{Preparation of Samples for Non-Targeted Metabolite Profiling and Analysis of GC/MS Profiles}

Dried down extracts were subjected to a two-step derivatization process using methoxyamine hydrochloride and $\mathrm{N}, \mathrm{O}$-bis(trimethylsilyl)trifluoroacetamide. Derivatized samples were injected splitless at $230{ }^{\circ} \mathrm{C}$ onto an Agilent 6890N GC equipped with a split/splitless inlet and a ZB-5 column (30 m $\times 0.25 \mathrm{~mm}, 0.25 \mu \mathrm{m} 95 \%$ dimethyl $/ 5 \%$ diphenyl polysiloxane film, $10 \mathrm{~m}$ integrated guard column, Phenomenex, Aschaffenburg, Germany). Eluting components were detected from $\mathrm{m} / \mathrm{z}$ 70-600 by using an Agilent 5975 Series Mass Selective Detector (Agilent Technologies, Waldbronn, Germany). For the generation of the metabolite profiles, chromatograms were baseline-corrected using Metalign [42]. Peak intensities above 500 arbitrary ion current units were imported into the TagFinder software [43], aligned using the retention index model of van den Dool and grouped according to their common retention time and mass spectral features. For statistical analysis, peak intensities of cluster (cluster size $>3$ ) were normalized to the internal standard (succinic acid-2,2,3,3- $\mathrm{d}_{4}$ ). Then, all data were $\log _{2}$-transformed and submitted to a two-sided Students $t$-test. Finally, resulting mass spectral features were identified via best mass spectral and retention index match using the Golm Metabolome Database [44] and the NIST2012 software (May 2011, National Institute of Standards and Technology, Gaithersburg, MD, USA). Details of the derivatization protocol and instrument parameters can be found in the Appendix C.

All statistical analysis was either performed with the $\mathrm{R}$ statistical language, the Bioconductor environment, the package pcaMethods or Microsoft Excel.

\subsection{Hormone Analysis}

Hormone profiling was conducted as described in Ziegler et al. [45] (for further information see Appendix D). Root material was homogenized, extracted in methanol, and processed firstly using a hydrophobic solid phase extraction cartridge (Chromabond Sorbent HR-XC, Macherey-Nagel, Düren, 
Germany) and secondly with an anion exchange solid phase extraction cartridge (Diethylaminoethyl Sephadex (DEAE-Sephadex)). For the root exudates the anion exchange step was omitted.

Analytes were separated by an Agilent 1290 Infinity HPLC system and detected on-line by ESI-MS/MS using an API 3200 triple-quadrupole LC-MS/MS system equipped with an ESI Turbo Ion Spray interface (AB Sciex, Darmstadt, Germany). Triple quadrupole scans were acquired in the multiple reaction monitoring mode (MRM) with Q1 and Q3 set at unit resolution. Scheduled MRM was performed with a window of $90 \mathrm{~s}$ and a target scan time of $0.1 \mathrm{~s}$. Selected MRM transitions and compound specific parameters can be found in Ziegler et al. [45].

\subsection{Microscope Images}

Bright-field and fluorescence microscopic images were recorded with a Stemi 2000 Axio Imager stereomicroscope (Carl Zeiss MicroImaging $\mathrm{GmbH}$, Göttingen, Germany). For bright-field images a Plan Apochromat $20 \times / 0.75$ objective with $20 \times$ magnification was used and for fluorescence images a Plan Apochromat $20 \times / 0.75$ objective with $20 \times$ magnification, a GFP-Filter $450-490 \mathrm{~nm}$, filterset 9 and the Axio Imager camera.

\subsection{Transcript Enrichment Analysis}

Overrepresentation analysis of the overexpressed genes in Arabidopsis 14 dpi as published in Lahrmann et al. [24] was performed with DAVID [46,47] against the default background genes from TAIR using KEGG pathways [48] and Gene Ontology [49].

\subsection{Data Availability}

All data sets are available from the MetaboLights repository [41] under the accession number MTBLS341.

\section{Conclusions}

The mutualistic interaction of $P$. indica with $A$. thaliana resulted in an increased shoot biomass production, but not root biomass after a two-week co-cultivation. Interestingly, the presence of $P$. indica had an obvious effect on the root's primary and secondary metabolism and the exudation rate, but not on leaf metabolism of $A$. thaliana. Apparently, $P$. indica stimulates the belowground metabolism of $A$. thaliana, but not the shoot metabolism. The metabolic changes identified can be considered as potential biomarkers, which need to be tackled in the near future. Previous studies and this study have shown that indolic glucosinolates and hormones are important for the interaction. The induction of the defense response might indicate that the plant tries to balance fungal growth and maintain its mutualism. This assumption could be confirmed by the analysis of appropriate mutants. In the future, new mutants, especially of the flavonoid metabolism, need to be obtained to investigate the mutualistic interaction in more depth. It is possible that plant-growth promoting microorganisms can be valuable tools for crop improvement $[7,50]$, as they promote the plant growth and help the plant to cope with abiotic and biotic stress factors.

Supplementary Materials: Supplementary materials can be found at http://www.mdpi.com/1422-0067/17/ 7/1091/s1.

Acknowledgments: This work was supported by the German Leibniz association (PAKT project 'Chemical Communication in the Rhizosphere'). The authors thank Steffen Neumann for upload of the data into MetaboLights and Alga Zuccaro for proofreading and the GFP-labeled P. indica strain. The publication of this article was funded by the Open Access fund of the Leibniz Association.

Author Contributions: Dierk Scheel and Nadine Strehmel conceived and designed the experiments; Siska Herklotz and Sylvia Krüger performed the experiments; Nadine Strehmel and Susann Mönchgesang analyzed the LC/MS and GC/MS data; Nadine Strehmel wrote the paper; Susann Mönchgesang and Dierk Scheel edited parts of the manuscript; Jörg Ziegler conducted the hormone profiling and proofread the manuscript. All authors have read and approved the final manuscript. 
Conflicts of Interest: The authors declare no conflict of interest.

\section{Abbreviations}

$\begin{array}{ll}\text { A. thaliana } & \text { Arabidopsis thaliana } \\ \text { P. indica } & \text { Piriformospora indica } \\ \text { GC } & \text { Gas chromatography } \\ \text { UPLC } & \text { Ultraperformance liquid chromatography } \\ \text { ESI } & \text { Electrospray ionisation } \\ \text { QTOF } & \text { Quadupole time of flight mass spectrometer } \\ \text { SD } & \text { Standard deviation } \\ \text { ET } & \text { Ethylene } \\ \text { JA } & \text { Jasmonic acid } \\ \text { GSL } & \text { Glucosinolate }\end{array}$

\section{Appendix A (Media for Co-Cultivation Studies)}

Cultivation of $P$. indica: For the cultivation of $P$. indica Complete Medium was used and prepared as follows: stock solution 1: $12 \%(w / v) \mathrm{NaNO}_{3}, 1.04 \%(w / v) \mathrm{KCl}, 1.04 \%(w / v) \mathrm{MgSO}_{4} \cdot 7 \mathrm{H}_{2} \mathrm{O}, 3.03 \%$ $(w / v) \mathrm{KH}_{2} \mathrm{PO}_{4}$ and stock solution 2: $0.6 \%(w / v) \mathrm{MnCl}_{2} \cdot 4 \mathrm{H}_{2} \mathrm{O}, 0.265 \%(w / v) \mathrm{ZnSO}_{4} \cdot 7 \mathrm{H}_{2} \mathrm{O}, 0.15 \%(w / v)$ $\mathrm{H}_{3} \mathrm{BO}_{3}, 0.075 \%(w / v) \mathrm{KI}, 0.025 \%(w / v) \mathrm{Na}_{2} \mathrm{MO}_{4} \cdot 2 \mathrm{H}_{2} \mathrm{O}, 0.013 \%(w / v) \mathrm{CuSO}_{4} \cdot 5 \mathrm{H}_{2} \mathrm{O}$. The final medium consisted of a mix of 5\% (v/v) stock solution 1, 2\% (w/v) Glucose, $0.2 \%(w / v)$ Bacto-Pepton, $0.1 \%(w / v)$ yeast extract, $0.1 \%(w / v)$ Casamino acids and $0.1 \%(v / v)$ stock solution 2.

Cultivation of $A$. thaliana and co-cultivation of $A$. thaliana with $P$. indica: For the pre- and co-cultivation stage $0.221 \%(w / v)$ Premix (M0231; Duchefa Biochemie B.V.) and 0.5\% $(w / v)$ sucrose in water were used. The $\mathrm{pH}$ was adjusted to 5.9 with $1 \mathrm{M} \mathrm{KOH}$ prior to autoclaving.

\section{Appendix B (UPLC/ESI-QTOFMS)}

C18-SPE: Bond Elut PPL cartridges were washed with $1 \mathrm{~mL}$ methanol, conditioned with $1 \mathrm{~mL}$ water/formic acid, 98/2 $(v / v)$, loaded with $4 \mathrm{~mL}$ sample solution, washed with $1 \mathrm{~mL}$ water, and eluted with $2 \mathrm{~mL}$ methanol/formic acid, 99/2 $(v / v)$; eluates were evaporated in a vacuum centrifuge and the residue were reconstituted in $120 \mu \mathrm{L}$ water/methanol, 70/30 (v/v).

Extraction of root material: $200 \mu \mathrm{L}$ methanol/water, $80 / 20(v / v)$, pre-cooled at $-28^{\circ} \mathrm{C}$, were added to the tissue; the mixture was allowed to reach room temperature within $15 \mathrm{~min}$ with occasionally vortexing; after sonication for $15 \mathrm{~min}$ at $20^{\circ} \mathrm{C}$ and centrifugation for $10 \mathrm{~min}$ at $16,000 \times g$ the supernatant was transferred to a new $2 \mathrm{~mL}$ polypropylene tube; the remaining plant material was extracted a second time with $200 \mu \mathrm{L}$ methanol/water, $80 / 20(v / v)$; both extracts were combined and evaporated to dryness at $40{ }^{\circ} \mathrm{C}$ using a vacuum centrifuge; the residue was redissolved in methanol/water, 30/70 $(v / v)$ according to fresh weight $(40 \mathrm{mg}=200 \mu \mathrm{L})$, sonicated for $10 \mathrm{~min}$ at $20^{\circ} \mathrm{C}$, centrifuged for $5 \mathrm{~min}$ at $16,000 \times g$, and the supernatant subjected to UPLC/ESI-QTOFMS analysis

UPLC settings: Full loop (loop volume: $2.5 \mu \mathrm{L}$ ); gradient: (flow rate: $150 \mu \mathrm{L} \cdot \mathrm{min}^{-1}$ ) $0-1 \mathrm{~min}$, isocratic $95 \% \mathrm{~A}, 5 \% \mathrm{~B} ; 1-16 \mathrm{~min}$, linear from $5 \%$ to $95 \% \mathrm{~B} ; 16-18 \mathrm{~min}$, isocratic $95 \% \mathrm{~B} ; 18-18.01 \mathrm{~min}$, linear from $95 \%$ to $5 \% \mathrm{~B} ; 18.01-20 \mathrm{~min}$, isocratic $5 \% \mathrm{~B}$.

ESI(+) settings: Nebulizer gas, nitrogen, $1.6 \mathrm{bar}$; dry gas, nitrogen, $6 \mathrm{~L} \cdot \mathrm{min}^{-1}, 190^{\circ} \mathrm{C}$; capillary, $-5000 \mathrm{~V}$; end plate offset, -500 V; funnel 1 RF, $200 \mathrm{Vpp}$; funnel 2 RF, $200 \mathrm{Vpp}$; in-source CID energy, $0 \mathrm{~V}$; hexapole RF, $100 \mathrm{Vpp}$; quadrupole ion energy, $3 \mathrm{eV}$; collision gas, argon; collision energy, $3 \mathrm{eV}$; collision RF $200 \mathrm{Vpp}$; transfer time, $70 \mu \mathrm{s}$; pre pulse storage, $5 \mu \mathrm{s}$; spectra rate, $3 \mathrm{~Hz}$.

ESI(-) settings: All parameters were maintained except for the nebulizer gas (1.4 bar), capillary (4000 V), quadrupole ion energy (5 eV), collision energy (7 eV), and collision RF (150 Vpp).

Data acquisition: centroid mode; recalibration on the basis of lithium formate cluster ions after injecting $20 \mu \mathrm{L} 10 \mathrm{mM}$ lithium hydroxide 49.9/49.9/0.2 (dissolved in isopropanol/water/formic acid; $v / v / v)$. 
XCMS settings: Feature detection with the help of the centWave algorithm (sntresh: 3, prefilter: (3.100), ppm: 25, peak width: (5.12); feature alignment with the help of the XCMS function group.density (minfrac: 0.75, bw: 2, mzwid: 0.05); missing values replacement by the XCMS function fillPeaks.

Analysis of raw data: DataAnalysis 4.2 software (Bruker Daltonics) for deconvolution and generation of extracted ion chromatograms

\section{Appendix C (GC/EI-QMS)}

Derivatization: Residues were reconstituted in $40 \mu \mathrm{L}$ methoxyaminehydrochloride $(20 \mathrm{mg} / \mathrm{mL}$ in pyridine, Sigma-Aldrich), the solution thoroughly vortexed and incubated at $40{ }^{\circ} \mathrm{C}$ for $1.5 \mathrm{~h}$. An $80 \mu \mathrm{L}$ mix comprising $70 \mu \mathrm{L} \mathrm{N,O-bis(trimethylsilyl)trifluoroacetamide} \mathrm{(BSTFA,} \mathrm{Macherey-Nagel)}$ and $10 \mu \mathrm{L}$ alkane reference mixture (dodecane, pentadecane, nonadecane, docosane, octacosane and dotriacontane each to a final concentration of $80 \mu \mathrm{g} \cdot \mathrm{mL}^{-1}$ dissolved in pyridine) were added and incubation at $40{ }^{\circ} \mathrm{C}$ proceeded for an additional $30 \mathrm{~min}$; the solution was centrifuged and the supernatant transferred to a GC vial.

GC settings: Carrier gas helium, constant flow: $1 \mathrm{~mL} \cdot \mathrm{min}^{-1}$; temperature program: $70^{\circ} \mathrm{C}$ for $1 \mathrm{~min}$, gradient $9 \mathrm{~K} \cdot \mathrm{min}^{-1}$ to $300^{\circ} \mathrm{C}, 5 \mathrm{~min}$ at $300^{\circ} \mathrm{C}$.

EI settings: Transfer line $300^{\circ} \mathrm{C}$; ion source temperature $230^{\circ} \mathrm{C}$; scan rate $3 \mathrm{~Hz}$

Metalign settings: Maximum amplitude: 6,000,000, peak slope factor: 0.5 , peak threshold factor: 1 , average peak width at half height: 5 .

TagFinder settings: Peak Finder (Smooth Width Apex Finder: 3; Low Intensity Threshold: 500; Max: Merging Time Width: 0.3); Time Scanner (Time Scan Width: 1; Min Fragment Intensity: 500); Tag Gen Filter (Tag Mass: 76, 146, 150-600; Sample Counts > 5); Intensity Calculator (Simple: MAX_INTENSITY); Tag Correlation (Correlation Method: PearsonCor; Significance Level: SIG_005); Tag Clustering (Core Adjacency Option: SAME_CORE; Min Core Option: INPUT_VALUE); Tag Output (Min Cluster Size: 3)

\section{Appendix D (Hormone Analysis)}

\section{Profiling of Root Tissue}

Homogenization and extraction: Root material was homogenized in bead beater and extracted with; $200 \mu \mathrm{L}$ methanol (supplemented with $2 \mathrm{ng}$ abscisic acid- $\mathrm{d}_{6}\left(\right.$ ABA- $\mathrm{d}_{6}$ ), $5 \mathrm{ng}$ indole-3-acetic acid- ${ }^{13} \mathrm{C}_{6}$ (IAA- $\left.{ }^{13} \mathrm{C}_{6}\right), 5 \mathrm{ng}$ jasmonic acid- $\mathrm{d}_{6}\left(\mathrm{JA}-\mathrm{d}_{6}\right), 0.74 \mathrm{ng}$ jasmonyl isoleucine- $\mathrm{d}_{2}$ (JA-Ile- $\left.\mathrm{d}_{2}\right)$, $30 \mathrm{ng}$ 12-oxo phytodienoic acid (OPDA- $\left.\mathrm{d}_{5}\right), 1.5 \mathrm{ng}$ salicylic acid- $\mathrm{d}_{4}\left(\mathrm{SA}-\mathrm{d}_{4}\right), 5 \mathrm{ng}$ zeatin $\left(\mathrm{Z}-\mathrm{d}_{5}\right)$, $5 \mathrm{ng}$ trans-zeatin-riboside- $\mathrm{d}_{5}\left(\mathrm{tZ}\right.$ ( $\left.\mathrm{R}-\mathrm{d}_{5}\right), 5 \mathrm{ng}$ dihydrozeatin riboside- $\mathrm{d}_{5}\left(\right.$ DHZR- $\left.\mathrm{d}_{5}\right)$. After vortexing for $20 \mathrm{~min}$ the supernatant was clarified by two rounds of centrifugation at 10,000 rpm for $5 \mathrm{~min}$. Before loading on the HR-XC SPE $1 \mathrm{~mL}$ water/acetic acid, 98/2 (v/v) was added.

HR-XC: The resin was conditioned with $1 \mathrm{~mL}$ methanol followed by $1 \mathrm{~mL}$ water (the liquid was passed through SPE 96 well plate ( $50 \mathrm{mg}$ HR-XC resin per well) by centrifugation at $250 \times g$ for $5 \mathrm{~min}$ using a JS5.3 bucket rotor in an Avanti J-26XP centrifuge (Beckman). Samples were transferred to the SPE 96 well plate, the resin washed with $1 \mathrm{~mL} \mathrm{H}_{2} \mathrm{O}$. Analytes were eluted successively by adding $1 \mathrm{~mL} \mathrm{MeOH}$ (for acidic hormones) and $1 \mathrm{~mL}$ methanolic ammonia $(0.35 \mathrm{M})$ for zeatins.

DEAE-Sephadex: The resin was washed with $1 \mathrm{~mL}$ methanol. The methanolic eluates from the HR-XC plates were loaded onto DEAE-Sephadex (acetate form, $50 \mathrm{mg} \cdot$ well $^{-1}$ ) filled. After washing with $1 \mathrm{~mL}$ methanol, the analytes were eluted with $1 \mathrm{~mL}$ of $3 \mathrm{M}$ acetic acid in methanol.

Further processing: eluates were transferred to $2 \mathrm{~mL}$ Eppendorf tubes and evaporated to dryness; residues were dissolved in $40 \mu \mathrm{L}$ of $20 \%(v / v)$ methanol, diluted with $40 \mu \mathrm{L}$ of water and centrifuged at $10,000 \times g$ for $10 \mathrm{~min}$. 
LC: Agilent 1290 Infinity HPLC; Nucleoshell RP18 column $(50 \times 3 \mathrm{~mm}$, particle size $2.7 \mu \mathrm{m}$; Macherey-Nagel, Düren, Germany) at $30{ }^{\circ} \mathrm{C}$; eluent (A: water $/ 0.2 \%(v / v)$ acetic acid; B: acetonitrile $/ 0.2 \%(v / v)$ acetic acid); flow rate: $0.5 \mathrm{~mL} \cdot \mathrm{min}^{-1}$; gradient for cytokinins: $2 \% \mathrm{~B}$ for $0.5 \mathrm{~min}$, followed by a linear increase to $28 \%$ B within $3 \mathrm{~min}$; increase to $98 \%$ in $0.5 \mathrm{~min}$ followed by an isocratic period of $1.5 \mathrm{~min}$ at $98 \% \mathrm{~B}$, starting conditions restored within the next $0.5 \mathrm{~min}$, and the column was allowed to re-equilibrate for $1 \mathrm{~min}$ at $2 \% \mathrm{~B}$; gradient for acidic phytohormones: $\mathrm{B}$ increased from $10 \%$ to $80 \%$ within $9 \mathrm{~min}$ after an initial hold at $10 \%$ B for $0.5 \mathrm{~min}$; further increase to $98 \% \mathrm{~B}$ within $0.5 \mathrm{~min}$; isocratic period at $98 \%$ B for $1.5 \mathrm{~min}$; column re-equilibrated at $10 \%$ B for $1 \mathrm{~min}$.

ESI(+) for cytokinins: curtain gas $50 \mathrm{psi}$, ion spray voltage $3500 \mathrm{~V}$, ion source temperature $650{ }^{\circ} \mathrm{C}$, nebulizing and drying gas 70 psi and 50 psi.

ESI(-) for acidic phytohormones: negative ion mode curtain gas $50 \mathrm{psi}$, ion spray voltage $-4500 \mathrm{~V}$, ion source temperature $350^{\circ} \mathrm{C}$, nebulizing and drying gas 70 psi and 50 psi.

Data evaluation: Peak areas were calculated automatically using the IntelliQuant algorithm of the Analyst 1.6.2 software (AB Sciex, Darmstadt, Germany) and manually supervised. All other calculations were performed with Excel (Microsoft Office Professional Plus 2010).

\section{Profiling of Root Exudates}

Sample preparation: Exudates were processed according to LC/MS-based metabolite profiling protocol; the residues were reconstituted in $200 \mu \mathrm{L}$ methanol (supplemented with $0.5 \mathrm{ng}$ ABA- $\mathrm{d}_{6}$, 2.5 ng IAA- ${ }^{13} \mathrm{C}_{6}, 1 \mathrm{ng} \mathrm{JA}-\mathrm{d}_{6}, 0.1 \mathrm{ng}$ JA-Ile- $\mathrm{d}_{2}, 4 \mathrm{ng}$ OPDA-d $\mathrm{d}_{5}, 0.4 \mathrm{ng}$ SA, $2.5 \mathrm{ng}$ Z-d $\mathrm{d}_{5}, 2.5 \mathrm{ng}$ tZ9R-d $\mathrm{d}_{5}$, $2.5 \mathrm{ng}$ DHZR- $\mathrm{d}_{5}$ ); incubated for $15 \mathrm{~min}$ at room temperature; after centrifugation, the supernatant was processed as described for root extracts, except for the omission of the DEAE-Sephadex SPE.

\section{References}

1. Verma, S.; Varma, A.; Rexer, K.H.; Hassel, A.; Kost, G.; Sarbhoy, A.; Bisen, P.; Bütehorn, B.; Franken, P. Piriformospora indica, gen. et sp. nov., a new root-colonizing fungus. Mycologia 1998, 90, 896-903. [CrossRef]

2. Weiss, M.; Selosse, M.A.; Rexer, K.H.; Urban, A.; Oberwinkler, F. Sebacinales: A hitherto overlooked cosm of heterobasidiomycetes with a broad mycorrhizal potential. Mycol. Res. 2004, 108, 1003-1010. [CrossRef] [PubMed]

3. Varma, A.; Bakshi, M.; Lou, B.; Hartmann, A.; Oelmueller, R. Piriformospora indica: A Novel Plant Growth-Promoting Mycorrhizal Fungus. Agric. Res. 2012, 1, 117-131. [CrossRef]

4. Sun, C.; Shao, Y.; Vahabi, K.; Lu, J.; Bhattacharya, S.; Dong, S.; Yeh, K.-W.; Sherameti, I.; Lou, B.; Baldwin, I.T.; et al. The beneficial fungus Piriformospora indica protects Arabidopsis from Verticillium dahliae infection by downregulation plant defense responses. BMC Plant Biol. 2014, 14, 268. [CrossRef] [PubMed]

5. Daneshkhah, R.; Cabello, S.; Rozanska, E.; Sobczak, M.; Grundler, F.M.W.; Wieczorek, K.; Hofmann, J. Piriformospora indica antagonizes cyst nematode infection and development in Arabidopsis roots. J. Exp. Bot. 2013, 64, 3763-3774. [CrossRef] [PubMed]

6. Camehl, I.; Sherameti, I.; Seebald, E.; Johnson, J.M.; Oelmüller, R. Role of Defense Compounds in the Beneficial Interaction Between Arabidopsis thalina and Piriformospora indica. In Piriformospora indica: Sebacinales and Their Biotechnological Applications; Varma, A., Kost, G., Oelmüller, R., Eds.; Springer-Verlag Berlin Heidelberg: New York, NY, USA, 2013; Volume 33, pp. 239-250.

7. Gill, S.S.; Gill, R.; Trivedi, D.K.; Anjum, N.A.; Sharma, K.K.; Ansari, M.W.; Ansari, A.A.; Johri, A.K.; Prasad, R.; Pereira, E.; et al. Piriformospora indica: Potential and Significance in Plant Stress Tolerance. Front. Microbiol. 2016, 7, 332. [CrossRef] [PubMed]

8. Das, A.; Kamal, S.; Shakil, N.A.; Sherameti, I.; Oelmueller, R.; Dua, M.; Tuteja, N.; Johri, A.K.; Varma, A. The root endophyte fungus Piriformospora indica leads to early flowering, higher biomass and altered secondary metabolites of the medicinal plant, Coleus forskohlii. Plant Signal. Behav. 2012, 7, 103-112. [CrossRef] [PubMed]

9. Prasad, R.; Kamal, S.; Sharma, P.K.; Oelmueller, R.; Varma, A. Root endophyte Piriformospora indica DSM 11827 alters plant morphology, enhances biomass and antioxidant activity of medicinal plant Bacopa monniera. J. Basic Microbiol. 2013, 53, 1016-1024. [CrossRef] [PubMed] 
10. Varma, A.; Verma, S.; Sudha; Sahay, N.; Butehorn, B.; Franken, P. Piriformospora indica, a cultivable plant-growth-promoting root endophyte. Appl. Environ. Microbiol. 1999, 65, 2741-2744. [PubMed]

11. Oelmueller, R.; Sherameti, I.; Tripathi, S.; Varma, A. Piriformospora indica, a cultivable root endophyte with multiple biotechnological applications. Symbiosis 2009, 49, 1-17. [CrossRef]

12. Yadav, V.; Kumar, M.; Deep, D.K.; Kumar, H.; Sharma, R.; Tripathi, T.; Tuteja, N.; Saxena, A.K.; Johri, A.K. A phosphate transporter from the root endophytic fungus Piriformospora indica plays a role in phosphate transport to the host plant. J. Biol. Chem. 2010, 285, 26532-26544. [CrossRef] [PubMed]

13. Bakshi, M.; Vahabi, K.; Sherameti, I.; Oelmuller, R.; Bhattacharya, S.; Baldwin, I.; Varma, A.; Yeh, K.-W.; Johri, A.K. WRKY6 restricts Piriformospora indica-stimulated and phosphate-induced root development in Arabidopsis. BMC Plant Biol. 2015, 15, 305. [CrossRef] [PubMed]

14. Kumar, M.; Yadav, V.; Kumar, H.; Sharma, R.; Singh, A.; Tuteja, N.; Johri, A.K. Piriformospora indica enhances plant growth by transferring phosphate. Plant Signal. Behav. 2011, 6, 723-725. [CrossRef] [PubMed]

15. Lahrmann, U.; Ding, Y.; Banhara, A.; Rath, M.; Hajirezaei, M.R.; Doehlemann, S.; von Wiren, N.; Parniske, M.; Zuccaro, A. Host-related metabolic cues affect colonization strategies of a root endophyte. Proc. Natl. Acad. Sci. USA 2013, 110, 13965-13970. [CrossRef] [PubMed]

16. Vahabi, K.; Sherameti, I.; Bakshi, M.; Mrozinska, A.; Ludwig, A.; Reichelt, M.; Oelmuller, R. The interaction of Arabidopsis with Piriformospora indica shifts from initial transient stress induced by fungus-released chemical mediators to a mutualistic interaction after physical contact of the two symbionts. BMC Plant Biol. 2015, 15, 58. [CrossRef] [PubMed]

17. Matsuo, M.; Johnson, J.M.; Sherameti, I.; Hieno, A.; Tokizawa, M.; Yamamoto, Y.Y.; Nomoto, M.; Tada, Y.; Godfrey, R.; Obokata, J.; et al. High REDOX RESPONSIVE TRANSCRIPTION FACTOR1 Levels Result in Accumulation of Reactive Oxygen Species in Arabidopsis thaliana Shoots and Roots. Mol. Plant 2015, 8, 1253-1273. [CrossRef] [PubMed]

18. Rafiqi, M.; Jelonek, L.; Akum, N.F.; Zhang, F.; Kogel, K.-H. Effector candidates in the secretome of Piriformospora indica, a ubiquitous plant-associated fungus. Front. Plant Sci. 2013, 4, 228. [CrossRef] [PubMed]

19. Vadassery, J.; Oelmuller, R. Calcium signaling in pathogenic and beneficial plant microbe interactions what can we learn from the interaction between Piriformospora indica and Arabidopsis thaliana. Plant Signal. Behav. 2009, 4, 1024-1027. [CrossRef] [PubMed]

20. Johnson, J.M.; Oelmueller, R. Agony to harmony-what decides? calcium signaling in beneficial and pathogenic plant-fungus interactions-what we can learn from the Arabidopsis/Piriformospora indica symbiosis. Mol. Microb. Ecol. Rhizosphere 2013, 2, 833-850.

21. Camehl, I.; Sherameti, I.; Venus, Y.; Bethke, G.; Varma, A.; Lee, J.; Oelmueller, R. Ethylene signalling and ethylene-targeted transcription factors are required to balance beneficial and nonbeneficial traits in the symbiosis between the endophytic fungus Piriformospora indica and Arabidopsis thaliana. New Phytol. 2010, 185, 1062-1073. [CrossRef] [PubMed]

22. Camehl, I.; Oelmueller, R. Do ethylene response factors- 9 and -14 repress PR gene expression in the interaction between Piriformospora indica and Arabidopsis? Plant Signal. Behav. 2010, 5, 932-936. [CrossRef] [PubMed]

23. Vadassery, J.; Ritter, C.; Venus, Y.; Camehl, I.; Varma, A.; Shahollari, B.; Novak, O.; Strnad, M.; Ludwig-Mueller, J.; Oelmueller, R. The role of auxins and cytokinins in the mutualistic interaction between Arabidopsis and Piriformospora indica. Mol. Plant Microbe Interact. 2008, 21, 1371-1383. [CrossRef] [PubMed]

24. Lahrmann, U.; Strehmel, N.; Langen, G.; Frerigmann, H.; Leson, L.; Ding, Y.; Scheel, D.; Herklotz, S.; Hilbert, M.; Zuccaro, A. Mutualistic root endophytism is not associated with the reduction of saprotrophic traits and requires a noncompromised plant innate immunity. New Phytol. 2015, 207, 841-857. [CrossRef] [PubMed]

25. Vahabi, K.; Camehl, I.; Sherameti, I.; Oelmueller, R. Growth of Arabidopsis seedlings on high fungal doses of Piriformospora indica has little effect on plant performance, stress, and defense gene expression in spite of elevated jasmonic acid and jasmonic acid-isoleucine levels in the roots. Plant Signal. Behav. 2013, 8, e26301. [CrossRef] [PubMed]

26. Nongbri, P.L.; Johnson, J.M.; Sherameti, I.; Glawischnig, E.; Halkier, B.A.; Oelmueller, R. Indole-3-acetaldoxime-derived compounds restrict root colonization in the beneficial interaction between Arabidopsis roots and the endophyte Piriformospora indica. Mol. Plant Microbe Interact. 2012, 25, 1186-1197. [CrossRef] [PubMed] 
27. Zuccaro, A.; Lahrmann, U.; Gueldener, U.; Langen, G.; Pfiffi, S.; Biedenkopf, D.; Wong, P.; Samans, B.; Grimm, C.; Basiewicz, M.; et al. Endophytic life strategies decoded by genome and transcriptome analyses of the mutualistic root symbiont Piriformospora indica. PLoS Pathog. 2011, 7, e1002290. [CrossRef] [PubMed]

28. Lahrmann, U.; Zuccaro, A. Opprimo ergo sum-evasion and suppression in the root endophytic fungus Piriformospora indica. Mol. Plant Microbe Interact. 2012, 25, 727-737. [CrossRef] [PubMed]

29. Böttcher, C.; Chapman, A.; Fellermeier, F.; Choudhary, M.; Scheel, D.; Glawischnig, E. The Biosynthetic Pathway of Indole-3-Carbaldehyde and Indole-3-Carboxylic Acid Derivatives in Arabidopsis. Plant Physiol. 2014, 165, 841-853. [CrossRef] [PubMed]

30. Lisec, J.; Schauer, N.; Kopka, J.; Willmitzer, L.; Fernie, A.R. Gas chromatography mass spectrometry-based metabolite profiling in plants. Nat. Protoc. 2006, 1, 387-396. [CrossRef] [PubMed]

31. Peskan-Berghoefer, T.; Shahollari, B.; Giong, P.H.; Hehl, S.; Markert, C.; Blanke, V.; Kost, G.; Varma, A.; Oelmueller, R. Association of Piriformospora indica with Arabidopsis thaliana roots represents a novel system to study beneficial plant-microbe interactions and involves early plant protein modifications in the endoplasmic reticulum and at the plasma membrane. Physiol. Plant. 2004, 122, 465-477. [CrossRef]

32. Peskan-Berghoefer, T.; Vilches-Barro, A.; Mueller, T.M.; Glawischnig, E.; Reichelt, M.; Gershenzon, J.; Rausch, T. Sustained exposure to abscisic acid enhances the colonization potential of the mutualist fungus Piriformospora indica on Arabidopsis thaliana roots. New Phytol. 2015, 208, 873-886. [CrossRef] [PubMed]

33. Cosme, M.; Wurst, S.; Cosme, M.; Franken, P.; Cosme, M.; Lu, J.; Erb, M.; Lu, J.; Erb, M.; Stout, M.J.; et al. A fungal endophyte helps plants to tolerate root herbivory through changes in gibberellin and jasmonate signaling. New Phytol. 2016. [CrossRef] [PubMed]

34. Khatabi, B.; Schafer, P. Ethylene in mutualistic symbioses. Plant Signal. Behav. 2012, 7, 1634-1638. [CrossRef] [PubMed]

35. Schaefer, P.; Pfiffi, S.; Voll, L.M.; Zajic, D.; Chandler, P.M.; Waller, F.; Scholz, U.; Pons-Kuehnemann, J.; Sonnewald, S.; Sonnewald, U.; et al. Manipulation of plant innate immunity and gibberellin as factor of compatibility in the mutualistic association of barley roots with Piriformospora indica. Plant J. 2009, 59, 461-474. [CrossRef] [PubMed]

36. Vanholme, R.; Demedts, B.; Morreel, K.; Ralph, J.; Boerjan, W. Lignin biosynthesis and structure. Plant Physiol. 2010, 153, 895-905. [CrossRef] [PubMed]

37. Bilski, P.; Li, M.Y.; Ehrenshaft, M.; Daub, M.E.; Chignell, C.F. Vitamin B6 (pyridoxine) and its derivatives are efficient singlet oxygen quenchers and potential fungal antioxidants. Photochem. Photobiol. 2000, 71, 129-134. [CrossRef]

38. Komarova, N.Y.; Thor, K.; Gubler, A.; Meier, S.; Dietrich, D.; Weichert, A.; Suter Grotemeyer, M.; Tegeder, M.; Rentsch, D. AtPTR1 and AtPTR5 transport dipeptides in planta. Plant Physiol. 2008, 148, 856-869. [CrossRef] [PubMed]

39. Hause, B.; Schaarschmidt, S. The role of jasmonates in mutualistic symbioses between plants and soil-born microorganisms. Phytochemistry 2009, 70, 1589-1599. [CrossRef] [PubMed]

40. Smith, C.A.; Want, E.J.; O'Maille, G.; Abagyan, R.; Siuzdak, G. XCMS: Processing mass spectrometry data for metabolite profiling using nonlinear peak alignment, matching, and identification. Anal. Chem. 2006, 78, 779-787. [CrossRef] [PubMed]

41. MetaboLights. Available online: http://www.ebi.ac.uk/metabolights/MTBLS341 (accessed on 3 July 2016).

42. Lommen, A. MetAlign: interface-driven, versatile metabolomics tool for hyphenated full-scan mass spectrometry data preprocessing. Anal. Chem. 2009, 81, 3079-3086. [CrossRef] [PubMed]

43. Luedemann, A.; Strassburg, K.; Erban, A.; Kopka, J. TagFinder for the quantitative analysis of gas chromatography-mass spectrometry (GC-MS)-based metabolite profiling experiments. Bioinformatics 2008, 24, 732-737. [CrossRef] [PubMed]

44. Golm Metabolome Database. Available online: http://gmd.mpimp-golm.mpg.de/.

45. Ziegler, J.; Qwegwer, J.; Schubert, M.; Erickson, J.L.; Schattat, M.; Burstenbinder, K.; Grubb, C.D.; Abel, S. Simultaneous analysis of apolar phytohormones and 1-aminocyclopropan-1-carboxylic acid by high performance liquid chromatography/electrospray negative ion tandem mass spectrometry via 9-fluorenylmethoxycarbonyl chloride derivatization. J. Chromatogr. A 2014, 1362, 102-109. [CrossRef] [PubMed]

46. DAVID Bioinformatics Resources 6.7. Available online: https://david.ncifcrf.gov/. 
47. Huang, D.W.; Sherman, B.T.; Lempicki, R.A. Systematic and integrative analysis of large gene lists using DAVID bioinformatics resources. Nat. Protoc. 2008, 4, 44-57. [CrossRef] [PubMed]

48. Kanehisa, M.; Goto, S. KEGG: Kyoto Encyclopedia of Genes and Genomes. Nucleic Acids Res. 2000, 28, 27-30. [CrossRef] [PubMed]

49. Ashburner, M.; Ball, C.A.; Blake, J.A.; Botstein, D.; Butler, H.; Cherry, J.M.; Davis, A.P.; Dolinski, K.; Dwight, S.S.; Eppig, J.T.; et al. Gene Ontology: tool for the unification of biology. Nat. Genet. 2000, 25, 25-29. [CrossRef] [PubMed]

50. Ansari, M.W.; Gill, S.S.; Tuteja, N. Piriformospora Indica a Powerful Tool for Crop Improvement. Proc. Indian Natl. Sci. Acad. 2014, 80, 317-324. [CrossRef]

(C) 2016 by the authors; licensee MDPI, Basel, Switzerland. This article is an open access article distributed under the terms and conditions of the Creative Commons Attribution (CC-BY) license (http:/ / creativecommons.org/licenses/by/4.0/). 